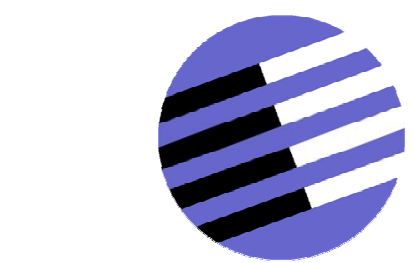

GOVERNANCE AND THE EFFICIENCY

OF ECONOMIC SYSTEMS

G ES Y

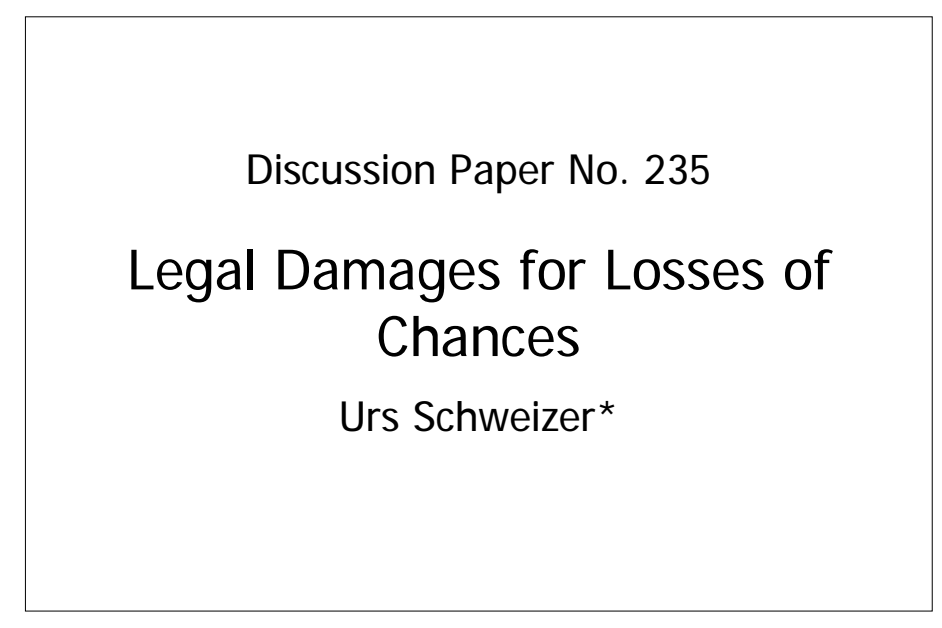

February 2008

*Urs Schweizer: Department of Economics, University of Bonn, Adenauerallee 24-42, 53113 Bonn, Germany, schweizer@uni-bonn.de

Financial support from the Deutsche Forschungsgemeinschaft through SFB/TR 15 is gratefully acknowledged. 


\title{
Legal Damages for Losses of Chances
}

\author{
Urs Schweizer, ${ }^{* \dagger}$ University of Bonn
}

February 22, 2008

\begin{abstract}
This paper deals with legal damages if losses of chances are at stake. In response to disparate ad hoc rules that have emerged from legal practice in Europe, the present paper proposes a unifying principle to handle such cases. Quite generally, the purpose of a damages award is to compensate the claimant and should be based on the difference in value between due performance and actual performance. To cope with limited observability, it is suggested to still award the difference though on average over the observed event. The paper calculates damages in line with this general principle. The proposed damage scheme is shown to fully compensate the victim and to provide efficient incentives for precaution, be it that multiple injurers act non-cooperatively or in concert, even if losses of chances are at stake.

JEL classification: K13, K12, D62

Keywords: estimating legal damages, liability for torts, liability for breach of contracts, uncertain causation, difference hypothesis

*address: Urs Schweizer, Department of Economics, University of Bonn, Adenauerallee 24, 53113 Bonn, Germany, schweizer@uni-bonn.de

$\dagger^{\dagger}$ The author thanks Alexander Stremitzer for helpful comments on a previous version of this paper. He also has benefitted from extensive discussions with Gerhard Wagner and other colleagues from the Law Department in Bonn. Support through SFB/TR 15 is
\end{abstract} gratefully acknowledged. 


\section{Introduction}

Three European courts were facing cases of the following type. A medical practitioner negligently provided a faulty diagnosis such that the correct treatment was postponed. Yet, even under correct treatment, the patient would have been cured with a probability of less than one. Should the practitioner be held liable and, if yes, to what extent? A court in England denied damages because, according to the court's view, the patient had failed to establish that the practitioner's wrongdoing has actually caused the loss. A court in Germany awarded full damages because, according to this court's view, the practitioner had failed to establish that his wrongdoing was not the cause of the patient's loss. A court in the Netherlands, finally, awarded damages amounting to that percentage of the patient's actual loss with which the patient would have been cured. ${ }^{1}$

Of a similar characteristic are cases involving attorneys whose clients, due to the attorney's negligent behavior, have lost at trial but would still have lost with a positive probability in the absence of the attorney's mistake. Again, courts in Europe tend to rule such cases in a rather nonuniform way. Worse, courts even in the same country apparently treat cases differently according to whether the negligent party happens to be a physician or an attorney. $^{2}$

Why is it so difficult to rule such cases and why are European courts coming up with rather disparate solutions in spite of the fact that the involved legal systems share central principles of damage rules? In fact, the purpose of a damages award for breach of contract (or for tort) is to compensate the claimant and should be based on the difference in value between the performance as provided and that agreed or, in tort cases, that resulting from meeting the negligence standard. In Germany, this basic principle is referred to as the difference hypothesis.

Conceptual difficulties possibly arise with the difference hypothesis as the actual situation under the party's negligent decision must be compared with the purely hypothetical one that would have resulted if, ceteris paribus, the party had met his obligation. Yet, what exactly does ceteris paribus mean if

\footnotetext{
${ }^{1}$ For details, the reader may wish to consult Mäsch (2004).

${ }^{2}$ See Mäsch (2004) again.
} 
the loss would still have occurred with positive probability even under proper treatment? In such cases, the exact difference between the actual and the hypothetical value remains uncertain.

The major piece of work on the determination of civil liability under such uncertainty is due to Shavell (1985). ${ }^{3}$ On efficiency grounds, Shavell argues in favor of liability in proportion to the probability of causation. His approach requires the assumption that each accident - an event involving a loss of wealth - is caused by exactly one entity. An entity may be one of several potential human injurers or it may be the "natural agent". In contrast, the present paper takes into account that causation could also be of a more interactive nature as the following stylized setting illustrates.

The victim consumes three randomly chosen units of a good, each of which possibly contains a toxic factor. If two or less of the units contain the toxic factor no harm occurs to the victim. Yet, if all three units (or more) are toxic the victim suffers from harm equivalent to, say, 90 units of wealth.

Imagine, by picking three units at random, the victim was hapless enough ending up with three toxic units. Suppose two units were supplied by injurer 1 , the third one by injurer 2 . As a consequence, the victim's wealth is actually reduced by 90 units of wealth. Yet, none of the entities can be said to have caused the accident entirely on its own. Rather, causation is of a more interactive nature and courts would probably hold the two injurers liable in proportion to their contribution of toxic factors for 60 and 30 units of wealth, respectively.

If it were just known that the victim has obtained three toxic units but the exact sources of these factors remain uncertain, courts tend to hold the two potential injurers liable in proportion of their market shares even if market shares may differ from the shares obtained by the particular victim at hand. Market shares are taken as a proxy for the unknown proportions. In fact, as will be shown in the paper, market shares can be interpreted as the expected value of the (unobservable) true proportions.

Let me now turn to the conceptually more difficult situation where one of the two entities is the "natural agent". The toxic factor may have invaded a unit as a consequence of the human injurer's negligence or, alternatively, it may have been added by nature. As a consequence, even in the absence of

\footnotetext{
${ }^{3}$ In contrast to the present study, Shavell's analysis refelcts the U.S. legal system.
} 
the human injurer, the victim may pick up three units containing the toxic factor. This fact must be faced when applying the difference hypothesis.

The actual situation is that the human injurer was present and the victim has picked up three toxic units. The hypothetical situation may be visualized by the following thought experiment. The victim would again have to pick up three units of the good at random but this time from a different box with a different composition of toxic and non-toxic units. If she picks up two or less toxic units the accidents is avoided in the hypothetical situation and, according to the difference hypothesis, correct legal damages would be equal to the full reduction of the victim's wealth. If, however, she picks up three toxic units as in the actual situation correct legal damages, according to the difference hypothesis, would be zero. Whether or not the accident is occurring in the hypothetical situation cannot be observed. The natural way out, again, would be to award legal damages in line with the difference hypothesis though, for lack of observability, on average over the observed event. In the example at hand, such average legal damages would be equal to the probability with which the accident were avoided in the hypothetical situation multiplied by the size of the loss.

Most economic studies of tort law - including Shavell (1985) - are departing from the accident model: A loss of fixed size $L$ is assumed to occur with a probability that depends on the level of precaution. By choosing insufficient precaution, the injurer has violated his obligation. Even at this negligent level, the loss would not have to occur for sure but suppose it did. Moreover, at the non-negligent level of precaution the accident would still occur with positive probability, say, $\varepsilon^{o}>0$.

The difference hypothesis requires to compare the actual situation with the hypothetical one that would have resulted from non-negligent precaution. At that point, the exact interpretation of the probability $\varepsilon^{o}$ matters. Suppose the fact that, under negligent precaution, an accident has occurred does not allow to update beliefs on whether or not an accident would also have occurred in the hypothetical situation where the injurer had met his obligation. Then, for the same reason as in the above example, legal damages on average over the observed event amount to $\left(1-\varepsilon^{o}\right) \cdot L$. Notice, these are exactly the damages, which the court in the Netherlands has awarded.

Alternatively, the probabilities of the accident model may refer to uncer- 
tainty that is type-contingent in the following sense. The probabilities are derived from relative frequencies of test series of cases that differ in type, which however injurers and courts are unable to distinguish. Nonetheless, the observed event - an accident has actually happened under negligent behavior - may allow to update the (hypothetical) probability with which the accident would still have happened if the injurer had met his obligation. Averaging out over the observed event would still be the doctrine to follow but would possibly lead to a different quantum of damages.

As an illustration of type-contingent uncertainty, consider the following setting which may mimic some of the malpractice cases though, of course, in a rather stylized way. Nature randomly selects one ball - the patient's type - out of a box containing known numbers of small, medium and large balls. Large balls reflect types that are cured even under improper treatment. Medium sized balls stand for patients that are cured but only under proper treatment. Small balls, finally, correspond to types that are never cured, not even if treatment was proper.

The injurer's negligence was to use, figuratively speaking, a coarsely meshed net letting pass all but big balls whereas his obligation consisted of using a finely woven net instead that would let pass only the small balls. If the net is letting pass a ball, the accident occurs. When deciding about the mesh size of his net, the injurer does not know the size of the ball chosen by nature. Notice, causation would be interactive again and could not be attributed uniquely to one of the entities.

Suppose an accident has actually occurred. Even if the exact size of the ball cannot be observed, the mere fact that an accident has occurred rules out that the ball was big. If it was of medium size then correct legal damages, according to the difference hypothesis, would be equal to the full loss whereas if it was small, correct legal damages would be zero as the use of a finely woven net would not have avoided the accident. Since the exact size of the ball, small or medium, is not known legal damages should be awarded on average over the observed event.

In this setting, ceteris paribus would mean that the random choice of nature is kept fixed when the actual situation, as a thought experiment, has to be compared with the hypothetical situation where the human injurer is using a finely woven net. As it turns out, in this setting, Shavell's proportionality 
rule emerges but with an interpretation that takes the interactive nature of causation into account.

The above examples indicate that a common doctrine - awarding correct legal damages on average over the observed event - allows to handle rather disparate situations. The quantum of damages emerging under this doctrine depends on the structure of the interaction between nature and injurers. Damages awarded according to the above doctrine compensate the victim for losses resulting from the injurer's deviation. Moreover, they provide efficient incentives provided that the injurer's obligation obeys the Hand Formula. These are the major findings of the paper, which is organized as follows.

Section 2 revisits the traditional accident model. The quantum of correct damages on average over observable events is explicitly determined. The examples capturing the contamination of victims as well as the one where nature selects the unobservable type of patients are spelled out in detail. Section 3 examines the more realistic situation involving losses of variable size. As it turns out, the approach based on the difference hypothesis easily extends to general distributions whereas Shavell's proportionality rule seems confined to the binary setting of the traditional accident model. Section 4 replaces the natural agent by a second human injurer who, in contrast to nature, may be held to share liability. An example is discussed where liability in proportion to market shares can be interpreted as taking averages over the observed event. Section 5 examines a general setting with multiple human injurers interacting with nature. It is shown that, from the efficiency perspective, details of how the injurers have to share liability do not matter. If the damage scheme obeys the Hand Formula, the victim is at least as well off as if the injurers had met their obligations and, by meeting his obligation, each injurer can unilaterally escape liability then the scheme provides efficient incentives for precaution, no matter whether the human injurers act noncooperatively or in concert. Section 6 concludes.

\section{The accident model revisited}

Party A is facing a decision $r \in R$ at $\operatorname{costs} c(r)$ that affects the value of party B's assets (B's wealth) in an uncertain way. An accident may or may not occur. If the accident occurs then B's wealth is reduced by a fixed amount 
$L$ whereas, if there is none, the value of B's assets stays constant. Moreover, it is known from test series of similar cases that the relative frequency of an accident amounts to $0 \leq \varepsilon(r) \leq 1$ provided that A has taken decision $r \in R$. If this test series consists of sufficiently many independent draws, $\varepsilon(r)$ can also be referred to as the probability of an accident.

The economic analysis of tort law refers to the setting at hand as the accident model. The model serves to investigate incentives for precaution arising from negligence rules. Suppose it were A's obligation to decide $r^{o} \in R$ but, instead, A has actually decided $r^{n} \neq r^{o} .{ }^{4}$ By such negligent behavior, A has saved private costs, i.e. $c^{n}=c\left(r^{n}\right)<c^{o}=c\left(r^{o}\right)$ but, at the same time, has raised the probability of an accident, i.e. $\varepsilon^{n}=\varepsilon\left(r^{n}\right)>\varepsilon^{o}=\varepsilon\left(r^{o}\right)$.

In order to specify the quantum of legal damages (if any), which A owes to $\mathrm{B}$, the actual situation must be compared with the hypothetical one that would have resulted if $\mathrm{A}$ had met his obligation. Conceptual difficulties arise as soon as the probability of an accident remains positive even if the obligation was met. In this case, meeting the obligation would not rule out the accident for sure but would merely improve the chances of avoiding it. What quantum of damages should be granted for the corresponding loss of a chance under negligent behavior?

To illustrate the issues at stake, the following example is considered, which captures the contamination of a victim from uncertain sources in a stylized way. The toxic factors are supplied by party A or, alternatively, by nature. Under negligent behavior, $K^{n}$ toxic among a total of $I^{n}+K^{n}$ units are around. If A had met his obligation, still $K^{o}<K^{n}$ toxic among a total of $I^{o}+K^{o}$ units would be around. In the actual (as well as the hypothetical) situation, party $\mathrm{B}$ is assumed to draw a sample of size $H$ at random. If his sample happens to contain $k^{c}$ toxic units or more, B's wealth is reduced by $L$. If it contains less, his wealth is not affected. The probabilities of accidents can easily be obtained from the hypergeometric distribution. If $K^{o}>k^{c}$ (as I assume) then the probability of an accident remains positive indeed, even if A had met his obligation.

Suppose it is known that, by choosing $r^{n}$, party A has neglected his obligation and an accident has actually occurred, i.e. party B's wealth is

\footnotetext{
${ }^{4}$ Instead of a tort relationship, the obligation may also arise from a contractual relationship.
} 
reduced by $L$. According to the difference hypothesis, the actual situation must be compared with the hypothetical one where A had met his obligation. Notice, in the hypothetical situation, party B would have to draw, by a thought experiment, another sample of size $H$, which would contain $k$ toxic units with probability

$$
\pi_{k}^{o}=\frac{\left(\begin{array}{c}
K^{o} \\
k
\end{array}\right) \cdot\left(\begin{array}{c}
I^{o} \\
H-k
\end{array}\right)}{\left(\begin{array}{c}
K^{o}+I^{o} \\
H
\end{array}\right)}
$$

and, hence, the probability that the accident would have been avoided in the hypothetical situation amounts to $1-\varepsilon^{o}=\sum_{k=0}^{k^{c}-1} \pi_{k}^{o}$. The correct quantum of legal damages should be $L$ if the hypothetical sample contains less than $k^{c}$ toxic elements, whereas it should be zero, if it contains (as in the actual situation) $k^{c}$ toxic elements or more. Therefore, on average, the quantum of legal damages would amount to $d=\left(1-\varepsilon^{o}\right) \cdot L$.

To make use of the difference hypothesis beyond the above example, the interaction between party $\mathrm{A}$ and nature must be expressed in what game theorists call normal form. ${ }^{5}$ From this perspective, nature is perceived as simultaneously "choosing" from a set $\omega \in \Omega$ of alternative moves - the outcome space - as party $\mathrm{A}$ is choosing from his set $r \in R$ of strategies. While $\mathrm{A}$ is assumed to behave strategically, nature is assumed to be governed by an exogenous probability measure $\pi$ : For any subset (event) $\Omega^{\prime}$ of the outcome space $\Omega, \pi\left(\Omega^{\prime}\right)$ denotes the probability, with which the event $\Omega^{\prime}$ occurs. The accident model in normal form combines this probability measure with a function $e: R \times \Omega \rightarrow\{0,1\}$, referred to as the accident technology. By construction, the accident technology attains the value $e(r, \omega)=1$ if and only if an accident is resulting from the interaction. In order to fit the data of the accident model,

$$
\varepsilon(r)=\operatorname{prob}\{\omega \in \Omega: e(r, \omega)=1\}
$$

must hold.

To begin with, suppose the actual move of nature $\omega$ is observable. Then legal doctrine would hold A fully liable for $L$ if the accident has actually occurred under A's negligent behavior but would have been avoided if A had

\footnotetext{
${ }^{5}$ Myerson (1999) attributes the important insight that any strategic interaction can be expressed in normal form to John von Neumann.
} 
met his obligation. In all other cases, A would not be held liable. For short, the quantum of damages awarded would amount to

$$
D(\omega)=\max \left[e\left(r^{n}, \omega\right)-e\left(r^{o}, \omega\right), 0\right] \cdot L,
$$

well in line with the difference hypothesis.

Notice, if a positive quantum of damages is awarded then $D(\omega)=\left[e\left(r^{n}, \omega\right)-\right.$ $\left.e\left(r^{o}, \omega\right)\right] \cdot L$ and the wealth position of party B is exactly the same as if party A had met his obligation. In other words, B is exactly compensated for the loss she suffers from A's deviation. If, however, the move of nature is such that the accident has actually been avoided but would have hypothetically occurred if A had met his obligation (i.e. $e\left(r^{n}, \omega\right)=0<e\left(r^{o}, \omega\right)=1$ ) then B enjoys a hypothetical windfall gain. It is common legal practice that B may keep such hypothetical windfall gains for free. As a consequence, B's ex ante expected wealth position $E\left[-e\left(r^{n}, \omega\right) \cdot L+D(\omega)\right]$ including compensation may exceed the ex ante expected wealth position $E\left[-e\left(r^{o}, \omega\right) \cdot L\right]$ if A had met his obligation. Legal scholars tend to dislike such overcompensation. Yet, let me emphasize that such overcompensation is not due to the quantum of damages as specified above but rather to the legal practice that hypothetical windfall gains are kept for free. It will be shown in section 5 below, that overcompensation, quite generally, does not distort incentives if party A's obligation obeys the Hand Formula.

This settles the issue of correct legal damages if the move of nature can be observed. Typically, however, the actual move of nature itself will not be observable. As a way out, correct legal damages should still be granted though on average over the observed event. More precisely, if the event $\Omega^{\prime} \subset \Omega$ is observed, average legal damages amount to

$$
d\left(\Omega^{\prime}\right)=E\left[D(\omega) \mid \Omega^{\prime}\right]
$$

and, by definition, are equal to the expected value of correct legal damages conditional on the observed event. Notice, if the outcome space is partitioned into observable events $\Omega=\Omega^{1} \cup \ldots \cup \Omega^{i} \cup \ldots \cup \Omega^{I}$ and if damages $d\left(\Omega^{i}\right)$ are granted in the event $\Omega^{i}$ then B's ex ante expected wealth position amounts to

$$
\sum_{i=1}^{I} \operatorname{prob}\left(\Omega^{i}\right) \cdot\left\{E\left[-e\left(r^{n}, \omega\right) \cdot L \mid \Omega^{i}\right]+d\left(\Omega^{i}\right)\right\}=E\left[-e\left(r^{o}, \omega\right) \cdot L+D(\omega)\right]
$$


as follows from Bayes' formula. In other words, from the ex ante perspective, it does not matter whether the moves of nature are observable or not. Incentives remain the same.

The focus of the present section will be on the event $\Omega_{a}=\{\omega \in \Omega$ : $\left.e\left(r^{n}, \omega\right)=1\right\}$ that an accident has actually occurred. This event is assumed to be observable. Suppose A has neglected his obligation and, indeed, an accident has occurred. For this event, windfall gains can be ruled out such that $D(\omega)=\left[e\left(r^{n}, \omega\right)-e\left(r^{o}, \omega\right)\right] \cdot L$ holds for any move of nature $\omega \in \Omega_{a}$. Therefore, from the perspective of the event $\Omega_{a}$, party $\mathrm{B}$ is exactly compensated for the loss from A's deviation.. Due to uncompensated windfall gains, party B's ex ante wealth position may still be strictly higher than if party A had met his obligation. In fact, let $\Omega_{w}=\left\{\omega \in \Omega: e\left(r^{n}, \omega\right)=0\right.$ but $\left.e\left(r^{o}, \omega\right)=1\right\}$ denote the event of such windfall gains. In the event $\Omega_{w}$, B does not suffer from any harm and, obviously, cannot claim damages, i.e. $D(\omega)=0$. As mentioned before, overcompensation from the ex ante perspective is due to the practice that windfall gains may be kept for free and not to the proposed specification of damages.

As a final piece of notation, let $\gamma=$ prob $\Omega_{w}$ denote the probability of hypothetical windfall gains. Notice, in the introductory example, such windfall gains would occur with positive probability. Then the following proposition can be established.

Proposition 1 Legal damages on average over the event that an accident has occurred amount to

$$
d\left(\Omega_{a}\right)=\frac{\varepsilon^{n}-\varepsilon^{o}+\gamma}{\varepsilon^{n}} \cdot L
$$

and can be calculated from the statistical data if, in addition, the probability $\gamma$ of hypothetical windfall gains is known.

Proof. Consider the partition $\Omega=\Omega_{a} \cup \Omega_{0} \cup \Omega_{w}$ of the outcome space where

$$
\Omega_{0}=\left\{\omega \in \Omega: e\left(r^{n}, \omega\right)=e\left(r^{o}, \omega\right)=0\right\} .
$$

It then follows from Bayes' rule and from consistency with the statistical data that

$$
\begin{aligned}
{\left[\varepsilon^{n}-\varepsilon^{o}\right] \cdot L } & =E\left[e\left(r^{n}, \omega\right)-e\left(r^{o}, \omega\right)\right] \cdot L= \\
& =\pi\left(\Omega_{a}\right) \cdot E\left[e\left(r^{n}, \omega\right)-e\left(r^{o}, \omega\right) \mid \Omega_{a}\right] \cdot L-\pi\left(\Omega_{w}\right) \cdot L= \\
& =\varepsilon^{n} \cdot d\left(\Omega_{a}\right)-\gamma \cdot L
\end{aligned}
$$


from which the claim follows immediately.

Several conclusions can be drawn from this proposition. If meeting the obligation would avoid the accident for sure, i.e. $\varepsilon^{o}=0$ then windfall gains cannot occur and average legal damages amount to $d\left(\Omega_{a}\right)=L$. Similarly, if neglecting the obligation leads to an accident for sure, i.e. $\varepsilon^{n}=1$ then windfall gains would also not occur and average legal damages amount to $d\left(\Omega_{a}\right)=\left(1-\varepsilon^{o}\right) \cdot L$. In general, if windfall gains can be ruled out then average legal damages amount to

$$
d\left(\Omega_{a}\right)=\frac{\varepsilon^{n}-\varepsilon^{o}}{\varepsilon^{n}} \cdot L
$$

In extension of the introductory example, the accident model $\varepsilon(r)$ is said to exhibit pure uncertainty if the fact that an accident has occurred under negligent behavior does not allow updating of believes for the hypothetical situation. Under pure uncertainty, the probability of windfall gains amounts to $\gamma=\left(1-\varepsilon^{n}\right) \cdot \varepsilon^{o}$ and, except for degenerate cases, is positive indeed. Moreover, it follows from the above proposition that average damages amount to $d\left(\Omega_{a}\right)=\left(1-\varepsilon^{o}\right) \cdot L$ provided that the probabilities underlying the accident model reflect pure uncertainty.

Type-contingent uncertainty, in contrast, would be involved if the probability $\varepsilon(r)$ results from relative frequencies of draws from samples containing cases that differ in type. For illustration, recall the second setting of the introduction where nature is assumed to select one ball out of a box containing $N_{s}$ small, $N_{m}$ medium and $N_{b}$ big balls. The ex ante probability of nature picking a small ball is $\varepsilon^{o}=N_{s} /\left(N_{s}+N_{m}+N_{b}\right)$ and of picking a medium ball is $\varepsilon^{n}-\varepsilon^{o}=N_{m} /\left(N_{s}+N_{m}+N_{b}\right)$. Yet, if a coarsely meshed net was used and an accident has actually occurred then it is known that the ball cannot be of big size. The updated probability that it was a ball of medium size then amounts to $N_{m} /\left(N_{s}+N_{m}\right)=\left(\varepsilon^{n}-\varepsilon^{o}\right) / \varepsilon^{n}$ and, hence, average legal damages are equal to (1), in line with Shavell's proportionality rule but based on the justification that correct legal damages are awarded on average over the observed event.

At the other extreme, finally, where all accidents that occur if A has met his obligation are of the windfall type, i.e. $\gamma=\varepsilon^{o}$, legal damages on average over the observed event amount to $d\left(\Omega_{1}\right)=L$, in line with the traditional negligence rule as pioneered by Brown (1973). Yet, to justify the traditional 
negligence rule, the occurrence of windfall gains must be at its implausible extreme.

\section{General distributions of asset values}

The accident model with a fixed loss size is widely used as a workhorse for the economic analysis of tort law. In real cases, however, the size of losses may vary. In the present section, it is shown how the concept of damages on average over observable events can be extended to more general distributions of wealth.

Party $\mathrm{A}$ is still facing a decision $r \in R$ at $\operatorname{costs} c(r)$ The decision affects party B's wealth $v$, which attains finitely many values from range $Y=\left\{v_{0}=\right.$ $\left.0<\ldots<v_{i}<\ldots<v_{H}\right\}$. At decision $r \in R$, the value $v_{i} \in Y$ is attained with probability $f_{i}(r)$. The cumulative distribution is denoted by $F_{i}(r)=$ $\sum_{j=0}^{i} f_{j}(r)$. The expected wealth is equal to

$$
E[v \mid r]=\sum_{i=0}^{H} f_{i}(r) \cdot v_{i}=v_{H}-\sum_{i=0}^{H-1} F_{i}(r) \cdot\left(v_{i+1}-v_{i}\right) .
$$

The decision actually taken and the obligation are still denoted by $r^{n}$ and $r^{o}$, respectively. I assume first order stochastic dominance, i.e. $F_{i}\left(r^{n}\right) \geq F_{i}\left(r^{o}\right)$ for all $i$ with strict inequality for one $i$ at least. It then follows that B's expected wealth is higher if the obligation was met, i.e. $E\left[v \mid r^{o}\right]>E\left[v \mid r^{n}\right]$.

The following modification of the stylized example introduced in the previous section may serve as illustration: If party B's sample of size $H$ contains $k$ toxic units and $i=H-k$ non-toxic elements then her wealth is reduced to $v_{i}$. If the sample is drawn at random then the probabilities amount to

$$
f_{i}\left(r^{n}\right)=\frac{\left(\begin{array}{c}
K^{n} \\
H-i
\end{array}\right) \cdot\left(\begin{array}{c}
I^{n} \\
i
\end{array}\right)}{\left(\begin{array}{c}
K^{n}+I^{n} \\
H
\end{array}\right)} \text { and } f_{j}\left(r^{o}\right)=\frac{\left(\begin{array}{c}
K^{o} \\
H-j
\end{array}\right) \cdot\left(\begin{array}{c}
I^{o} \\
j
\end{array}\right)}{\left(\begin{array}{c}
K^{o}+I^{o} \\
H
\end{array}\right)}
$$

Suppose it is known that A has neglected his obligation and that B's actual wealth is $v_{i}$. To determine the quantum of damages due, her actual wealth $v_{i}$ must be compared with her hypothetical wealth $v_{j}$ if $\mathrm{A}$ had met his obligation. If this hypothetical wealth were known then the correct quantum of damages would amount to $D_{i j}=\max \left[v_{j}-v_{i}, 0\right]$ as follows from the difference hypothesis. 
Typically, however, the hypothetical level of wealth cannot be observed. In this case, the correct quantum of damages should still be awarded though on average over the observed event. Since, in the hypothetical situation, party $\mathrm{B}$ would draw another sample at random the situation would correspond to one of pure uncertainty which means that $v$ attains the value $v_{j}$ with probability $f_{j}\left(r^{o}\right)$. If it is observed that the actual value under negligent behavior is $v_{i}$ then average damages amount to

$$
d_{i}=\sum_{j=i}^{H} f_{j}\left(r^{o}\right) \cdot\left(v_{j}-v_{i}\right) .
$$

To extend the setting beyond the above example, the interaction between party A and nature must again be expressed in normal form. Nature is assumed to contribute a random move $\omega \in \Omega$ where nature's choice is governed by the probability measure $\pi$. The wealth function $V: R \times \Omega \rightarrow Y$ yields the level of wealth $v=V(r, \omega)$ that results from the interaction between A's decision $r$ and nature's move $\omega$. To be consistent with the data,

$$
f_{i}(r)=\operatorname{prob}\left\{\omega \in \Omega: V(r, \omega)=v_{i}\right\}
$$

is assumed to hold.

Suppose it is known that party A, by choosing $r^{n} \neq r^{o}$, has neglected his obligation and that the move $\omega$ of nature is observed. Then correct legal damages, according to the difference hypothesis, would amount to

$$
D(\omega)=\max \left[V\left(r^{o}, \omega\right)-V\left(r^{n}, \omega\right), 0\right] .
$$

Party B's ex ante expected wealth position $E\left[V\left(r^{n}, \omega\right)+D(\omega)\right]$ including damages may again exceed the position $E\left[V\left(r^{o}, \omega\right)\right]$ resulting from A's meeting his obligation. Again, such overcompensation is due to the legal practice that hypothetical windfall gains are kept for free and not to the quantum of damages as specified above.

In most cases, however, the move of nature cannot be observed but suppose the actual value $v_{i}=V\left(r^{n}, \omega\right)$ is observable which means that the event

$$
\Omega_{i}=\left\{\omega \in \Omega: V\left(r^{n}, \omega\right)=v_{i}\right\}
$$

is observable. Correct damages on average over the observed event then amount to

$$
d\left(\Omega_{i}\right)=E\left[D(\omega) \mid \Omega_{i}\right]
$$


Notice, in contrast to the binary accident model examined in the previous section, party B may be strictly better off even from the perspective of the event $\Omega_{i}$. For such overcompensation to take place, hypothetical windfall gains must occur with positive probability, i.e.

$$
\operatorname{prob}\left\{\omega \in \Omega: V\left(r^{n}, \omega\right)=v_{i}>V\left(r^{o}, \omega\right)\right\}>0
$$

must hold. From the ex ante perspective, B's expected wealth position is the same as if moves of nature were observable, i.e. it holds that

$$
E\left[V\left(r^{n}, \omega\right)+D(\omega)\right]=E\left[V\left(r^{n}, \omega\right)\right]+\sum_{i=1}^{n} \operatorname{prob}\left(\Omega_{i}\right) \cdot d\left(\Omega_{i}\right)
$$

If hypothetical windfall gains are ruled out, the correct quantum of damages can be calculated according to the following proposition.

Proposition 2 In the absence of hypothetical windfall gains, correct damages on average over the event $\Omega_{i}$ that $B$ 's actual wealth is $v_{i}$ amount to

$$
d\left(\Omega_{i}\right)=E\left[V\left(r^{o}, \omega\right) \mid \Omega_{i}\right]-v_{i}
$$

Proof. If windfall gains can be ruled out then $D(\omega)=V\left(r^{o}, \omega\right)-V\left(r^{n}, \omega\right)$ must hold for any move of nature. Since $E\left[V\left(r^{n}, \omega\right) \mid \Omega_{i}\right]=v_{i}$, claim (2) is obviously true.

The obvious generalization of Shavell's proportionality rule would be to grant damages according to (2) even in cases where hypothetical windfall gains cannot be ruled out. Yet, if the victim's wealth attains more than two values and if hypothetical windfall gains occur with positive probability this solution may possibly assign a negative quantum of damages. In this sense, a generalization of Shavell's proportionality rule (beyond the accident model with fixed loss size) generally leading to a non-negative quantum of damages is not available. ${ }^{6}$

\footnotetext{
${ }^{6}$ Notice, if party B's actual level of wealth cannot be observed, it would be possible to grant damages according to Kahan's (1989) rule which amount to $E\left[V\left(r^{o}, \omega\right)-V\left(r^{o}, \omega\right)\right]$. This means that, while taking averages over the observed event, windfall gains are offset against losses.
} 


\section{Two potential human injurers: an example}

So far, the interaction between one human party and nature has been examined. In the present section, nature is replaced by a second human player. Suppose both human injurers have neglected their obligations. As a consequence, there are $K^{n}=M_{1}+M_{2}$ toxic among a total of $K^{n}+I^{n}$ units around, out of which party B consumes a sample of size $H$ randomly drawn. $M_{q}$ denotes the total number of toxic units supplied by $q$. If party B's sample happens to contain $k^{c}$ toxic units or more then her wealth is reduced by the fixed amount $L$. Notice, in contrast to the example of section 2, nature does no longer contribute toxic factors. Nevertheless, a move of nature remains involved, which governs the random choice of the victim. I further assume that no toxic units were around and, as a consequence, the accident would have been avoided if both injurers had met their obligations.

What damages do the injurers owe to victim B? Most legal systems would grant the victim recovery of the loss resulting from the injurers' negligence. Put differently, the victim is treated as if a single party were responsible for the deviations. But in what proportions would the injurers be held liable?

To begin with, suppose it is known that B's sample contains $k=m_{1}+$ $m_{2} \geq k^{c}$ toxic units and that injurer $q$ has contributed $m_{q}$ of them. Then legal practice, quite likely, would have the injurers sharing damages in proportion to their contributions such that party $q$ would owe damages in the amount of

$$
D_{q}=\frac{m_{q}}{m_{1}+m_{2}} \cdot L
$$

to the victim $\mathrm{B}$. In particular, if it were known that all the toxic units came from the same injurer, say $m_{1}+m_{2}=m_{1}$ then injurer $q=1$ would have to bear the full loss on its own.

I now turn to the more interesting case where it is still known that victim B's sample actually contains $k \geq k^{c}$ toxic elements but the shares contributed by the two injures remain uncertain. In such a case, quite likely, courts would hold the injurers liable in proportion of their market shares such that party $q$ would now owe damages in the amount of

$$
D_{q}=\frac{M_{q}}{M_{1}+M_{2}} \cdot L
$$

to the victim B. It will be shown that market shares can be interpreted as 
expected or average proportions according to which the injurers have contributed toxic units to the victim's sample.

At the time courts are called in, the share of toxic units consumed by the particular victim at hand and contributed by injurer $q$ is not random any more, it is just not known. From the ex ante view, however, this share would be random. In fact, since the victim is visualized as drawing a sample of size $H$, the ex ante probability $p\left(m_{1}, m_{2}\right)$ that his sample will contain $m_{q}$ toxic elements contributed by injurer $q(q=1,2)$ amounts to

$$
p\left(m_{1}, m_{2}\right)=\frac{\left(\begin{array}{c}
M_{1} \\
m_{1}
\end{array}\right) \cdot\left(\begin{array}{c}
M_{2} \\
m_{2}
\end{array}\right) \cdot\left(\begin{array}{c}
I^{n} \\
H-m_{1}-m_{2}
\end{array}\right)}{\left(\begin{array}{c}
M_{1}+M_{2}+I^{n} \\
H
\end{array}\right)} .
$$

Similarly, the probability that the sample contains exactly $k$ toxic elements amounts to

$$
P(k)=\sum_{m=0}^{k} p(m, k-m)=\frac{\left(\begin{array}{c}
M_{1}+M_{2} \\
k
\end{array}\right) \cdot\left(\begin{array}{c}
I^{n} \\
H-k
\end{array}\right)}{\left(\begin{array}{c}
M_{1}+M_{2}+I^{n} \\
H
\end{array}\right)} .
$$

These ex ante probabilities may now be used to calculate average legal damages. If it is just known that the sample contains $k$ toxic elements then average damages owed by injurer $q=1$ amount to

$$
d_{1}(k)=\sum_{m=0}^{k} \frac{p(m, k-m)}{P(k)} \cdot \frac{m}{k} \cdot L .
$$

The following proposition establishes that average damages in the above sense are identical with liability in proportion to market shares.

Proposition 3 Suppose ex ante probabilities are used as weights to form averages. Damages in proportion to market shares (4) are then equal to correct damages (3) on average over the observed event.

Proof. The claim is established by the following calculation for injurer $q=1$ (for the other injurer, a similar calculation applies):

$$
\begin{aligned}
d_{1}(k) & =\sum_{m=0}^{k} \frac{\left(\begin{array}{c}
M_{1} \\
m
\end{array}\right) \cdot\left(\begin{array}{c}
M_{2} \\
k-m
\end{array}\right)}{\left(\begin{array}{c}
M_{1}+M_{2} \\
k
\end{array}\right)} \cdot \frac{m}{k} \cdot L \\
& =\sum_{m=1}^{k} \frac{\left(\begin{array}{c}
M_{1}-1 \\
m-1
\end{array}\right) \cdot\left(\begin{array}{c}
M_{2} \\
k-m
\end{array}\right)}{\left(\begin{array}{c}
M_{1}+M_{2} \\
k
\end{array}\right)} \cdot \frac{M_{1}}{k} \cdot L \\
& =\sum_{m=0}^{k-1} \frac{\left(\begin{array}{c}
M_{1}-1 \\
m
\end{array}\right) \cdot\left(\begin{array}{c}
M_{2} \\
k-1-m
\end{array}\right)}{\left(\begin{array}{c}
M_{1}+M_{2} \\
k
\end{array}\right)} \cdot \frac{M_{1}}{k} \cdot L
\end{aligned}
$$




$$
=\frac{\left(\begin{array}{c}
M_{1}+M_{2}-1 \\
k-1
\end{array}\right)}{\left(\begin{array}{c}
M_{1}+M_{2} \\
k
\end{array}\right)} \cdot \frac{M_{1}}{k} \cdot L=\frac{M_{1}}{M_{1}+M_{2}} \cdot L
$$

as was to be shown.

Notice, average damages owed by an injurer do not depend on the exact number $k$ of toxic elements consumed by the victim. Therefore, if it were just known that the victim's sample must have contained at least $k^{c}$ toxic units, average damages would still be in proportion to market shares.

The example could easily be extended by having nature also contributing toxic factors. Total damages owed by the injurers would then have to be calculated as in section 2 by taking averages over the observed event. The probabilities of the random draw in the hypothetical situation would have to serve as weights. These damages $d$ would then have to be shared according to the principles laid down in the present section. It can be shown that, under this scheme, injurer $q$ would owe damages amounting to

$$
d_{q}=\frac{M_{q}}{M_{1}+M_{2}} \cdot d
$$

Total damages $d$ awarded to the victim would differ from $L$ if nature also adds toxic factors but would still have to be borne in proportion to market shares.

\section{$5 \quad$ Multiple injurers and efficient incentives}

Up to now, the focus was on the quantum of damages to be awarded in line with the difference hypothesis. Concepts from microeconomic theory were used to settle conceptual issues concerning the difference hypothesis for situations where a reduction of a party's wealth cannot be ruled out, not even if all obligations were met. In this section, I turn to the more traditional turf of the economic analysis of law. The quantum of damages affects the incentives for precaution. It is shown that damages in line with the general principles laid down in the present paper provide efficient incentives if the obligations are consistent with the Hand Formula. To establish the efficiency result in general, the model of section 3 is extended to multiple injurers. ${ }^{7}$

\footnotetext{
${ }^{7}$ For a systematic discussion of multilateral obligations in general, the reader is referred to Schweizer (2005b).
} 
The class of potential injurers is denoted by $q=1, \ldots, Q$. Party $q$ is facing a decision $r_{q} \in R_{q}$ and bears costs $c_{q}\left(r_{q}\right)$. Profiles of decisions are denoted by

$$
r=\left(r_{1}, \ldots, r_{q}, \ldots, r_{Q}\right) \in R=R_{1} \times \ldots \times R_{q} \times \ldots \times R_{Q}
$$

and total costs by

$$
c(r)=\sum_{q=1}^{Q} c_{q}\left(r_{q}\right)
$$

Nature's contribution is captured by the random move $\omega \in \Omega$. The wealth $v=V(r, \omega)$ of party B resulting from the interaction attains values in the range $Y=\left\{v_{0}, \ldots, v_{H}\right\}$. Value $v_{i}$ is attained with probability $f_{i}(r)$ if the injurers have chosen profile $r \in R$.

Let $D_{q}(r, \omega)$ denote correct legal damages owed by party $q$ to party B if the injurers have chosen profile $r \in R$ and nature's move is $\omega \in \Omega$. Since compensating the victim for the injurers' negligence is at stake, party $B$ should be made as well off as if all the wrongdoings could be attributed to a single party. According to this principle,

$$
\sum_{q=1}^{Q} D_{q}(r, \omega)=D(r, \omega)
$$

must hold where total damages amount to

$$
D(r, \omega)=\max \left[V\left(r^{o}, \omega\right)-V(r, \omega), 0\right]
$$

as in section $3 .^{8}$ Under this principle, party B is at least as well off as if the injurers had met their obligations, i.e.

$$
V(r, \omega)+D(r, \omega)=V(r, \omega)+\sum_{q=1}^{Q} D_{q}(r, \omega) \geq V\left(r^{o}, \omega\right)
$$

must hold for any profile $r \in R .^{9}$

Suppose, by acting in concert and aiming at minimizing their total expenses, the injurers have agreed to profile $r^{c}$. Since one of their option would have been to meet their obligations, as a group, they cannot be worse off under the actions cooperatively chosen, i.e.

$$
-E\left[D\left(r^{c}, \omega\right)\right]-c\left(r^{c}\right) \geq-E\left[D\left(r^{o}, \omega\right)\right]-c\left(r^{o}\right)=-c\left(r^{o}\right)
$$

\footnotetext{
${ }^{8}$ Windfall gains do not occur if $V\left(r^{o}, \omega\right) \geq V\left(r^{n}, \omega\right)$ holds for all moves $\omega \in \Omega$ of nature. In this case, $D(r, \omega)=V\left(r^{o}, \omega\right)-V\left(r^{n}, \omega\right)$.

${ }^{9}$ This requirement corresponds to the saddle point property, which Schweizer (2005a) has identified as the driving force behind efficient incentives in general.
} 
must hold. But party B, under $r^{c}$, will also be at least as well off as under due performance. It follows that damages as specified above provide incentives to meet the normative content behind the obligation $r^{o}$.

In particular, if the obligation profile $r^{o}=\left(r_{1}^{o}, \ldots, r_{Q}^{o}\right) \in R$ obeys the (generalized) Hand Formula in the sense that it maximizes expected social surplus, i.e.

$E\left[V\left(r^{o}, \omega\right)\right]-c\left(r^{o}\right)=\sum_{i=0}^{H} f_{i}\left(r^{o}\right) \cdot v_{i}-c\left(r^{o}\right) \geq E[V(r, \omega)]-c(r)=\sum_{i=0}^{H} f_{i}(r) \cdot v_{i}-c(r)$

then it follows that

$$
\begin{aligned}
-E\left[D\left(r^{c}, \omega\right)\right]-c\left(r^{c}\right) & \leq-E\left[D\left(r^{c}, \omega\right)\right]+E\left[V\left(r^{o}, \omega\right)\right]-E\left[V\left(r^{c}, \omega\right)\right]-c\left(r^{o}\right) \\
& \leq-c\left(r^{o}\right) \leq-E\left[D\left(r^{c}, \omega\right)\right]-c\left(r^{c}\right) .
\end{aligned}
$$

Therefore, all of the above inequalities must be binding and, hence, the cooperatively chosen profile $r^{c}$ must be efficient. In other words, the injurers cannot improve their joint position, not even by deviating from their obligation in a collusive way.

Finally, injurers can also not improve their position unilaterally if the damage scheme satisfies one additional principle, namely no liability without negligence. More precisely, if each potential injurer, by unilaterally meeting his obligation, can escape liability, i.e.

$$
D_{q}\left(r_{q}^{o}, r_{-q}, \omega\right)=0
$$

for any profile $r_{-q}$ of the other injurers then all injurers meeting their obligations is a Nash equilibrium of the game induced by such a damage scheme as the following proposition establishes.

Proposition 4 If the injurers' obligations obey the Hand Formula and damages are awarded, which fully compensates the victim for the injurers' deviations then, by meeting all their obligations, the injurers minimize the sum of precaution costs and damages owed to B. If, in addition, no injurer who has met his obligation is held liable then all parties have the incentive to meet their obligations, i.e. $r^{o}$ is a Nash equilibrium of the game induced by such a damage rule. 
Proof. The first claim has already been established above. To establish the second claim, suppose all but injurer $q$ have met their obligations. It then follows from the first claim that

$$
c_{q}\left(r_{q}\right)+\sum_{p \neq q} c_{p}\left(r_{p}^{o}\right)+E\left[D\left(r_{q}, r_{-q}^{o}, \omega\right)\right] \geq c\left(r^{o}\right)=c_{q}\left(r_{q}^{o}\right)+\sum_{p \neq q} c_{p}^{o}\left(r_{p}\right)
$$

and, hence that

$$
c_{q}\left(r_{q}\right)+E\left[D_{q}\left(r_{q}, r_{-q}^{o}, \omega\right)\right] \geq c_{q}\left(r_{q}^{o}\right)
$$

must hold. The last inequality establishes that meeting his own obligation is a best response of injurer $q$ to all the other injurers meeting theirs and, hence, the profile $r^{o}$ must be a Nash equilibrium indeed.

Notice, for the same reason as in section 3, party B may be better off than if all the injurers had met their obligations. Nevertheless, incentives for overinvestment in precaution do not arise because, by meeting his obligation, each potential injurer avoids liability and, for that reason, has no incentives to invest beyond.

\section{Concluding remarks}

One or several human injurers have negligently deviated from their obligations, which has resulted in a reduction of the victim's wealth. What quantum of damages should be awarded to the victim and how should the potential injurers share liability? While many legal systems are making use of what, in Germany at least, is referred to as the difference hypothesis different courts, nevertheless, are coming up with rather disparate solutions in cases where losses of chances are at stake. To overcome such disorder, the present paper proposes - as a general principle - strictly to adhere to the difference hypothesis and, if observability is limited, to take averages over the observed event.

To implement the proposed scheme, probabilities as in the traditional accident model must be known. If these probabilities reflect pure uncertainty correct damages on average over the observed event can easily be determined. If, however, the probabilities are type-contingent but types cannot be observed then additional information would be needed.

In cases where several human injurers are involved rules are required of how they should share the burden of liability. In practical cases, equal shares 
of market shares or other indicators of the specific case may serve as measures ad hoc. As a plausible and generally available alternative, liability could also be assigned in proportion to an injurer's cost savings from neglecting his

obligation. In any case, from the efficiency viewpoint, the exact details of the arrangement do not matter. If the Hand Formula is met, the victim is treated as if the wrongdoings were due to a single party and the negligence principle is maintained then the damage scheme generates efficient incentives for precaution quite generally.

The present paper is extending principles, elsewhere widely accepted by legal practice, to damages for losses of chances. I am realist enough not to expect that legal practice will quickly jump at the conclusions drawn from a theoretical paper, particularly not if written by an economist. But, hopefully, I have shown that tools from microeconomic theory allow to disentangle some of the major issues at stake.

\section{References}

Brown, J. P. (1973), "Toward an Economic Theory of Liability", Journal of Legal Studies 2, 323 - 349.

Kahan, M. (1989): "Causation and incentives to take care under the negligence rule", Journal of Legal Studies, 18, 427 - 447.

Mäsch, G. (2004): Chance und Schaden. Mohr Siebeck: Tübingen.

Myerson, R. B. (1999), "Nash equilibrium and the history of economic theory", Journal of Economic Literature XXXVII, 1067 - 1082.

Schweizer, U. (2005a), "Law and Economics of Obligations," International Review of Law and Economics 25, 209 - 228.

Schweizer, U. (2005b), "The pure theory of multilateral obligations", Journal of Institutional and Theoretical Economics (JITE) 161, 239 - 254.

Shavell, S. (1985): "Uncertainty over causation and the determination of civil liability", The Journal of Law and Economics 28, 587 - 609. 